\title{
Rational exponential solutions of conformable space-time fractional equal-width equations
}

https://doi.org/10.1515/nleng-2018-0076

Received May 16, 2018; revised July 5, 2018; accepted August 3, 2018.

\begin{abstract}
In this paper, the rational exponential solutions of two space-time fractional equal-width (FEW) equations are explored in the conformable derivative sense. The way to reach explicit exact solutions is to transform the fractional order PDEs into a nonlinear ODEs of discrete order through some properties of conformable derivatives and a fractional complex transforms. The subsequent equations have been elucidated by employing the $\exp _{a}$ function approach. Some new exact solutions of the said equations are effectively formulated and graphically conveyed with the aid of symbolic computation in Mathematica and MATLAB respectively.
\end{abstract}

Keywords: Space-time fractional equal-width equations; Conformable derivatives; Exp a function approach; Rational exponential solutions

\section{Introduction}

Various fields of science and engineering are influenced by Leibnitz's work on fractional calculus and having increasing impact on these sciences during last twenty years ostensibly $[1,2]$. Many definitions of fractional derivatives, Like Hilfer, Riemann-Liouville, Caputo form and so on, have been introduced in the literature. Here, in this paper, we are interested in making the use of a recent definition of fractional derivatives, called conformable fractional derivatives given by Khalil et al. [3]. The exact solutions have always been a particular importance among the researchers in many fields of nonlinear sciences. The availability of symbolic computation softwares is a direct help to minimize the manual labor for finding problematic solutions to nonlinear evolution equations. Various analytical methods have been presented in the literature to explore exact solutions such as ansatz [4, 5], modified sim-

\footnotetext{
*Corresponding Author: Asim Zafar, Department of Mathematics, COMSATS University Islamabad, Vehari Campus, Pakistan, E-mail: asimzafar@hotmail.com
}

ple equation $[6,7]$, the extended trial equation $[8,9]$, the first integral [10, 11], $\left(\frac{G^{\prime}}{G}\right)$-expansion [12], sine-Gordon expansion $[13,14]$. Furthermore, some other excellent works like generalized Kudryashov method [15-17], a modified form of Kudryashov and functional variable methods [1822] have been done by different researchers. In [23-28], the auxiliary equation, the extended tanh-function, the improved $\tan \left(\frac{\phi(\eta)}{2}\right)$-expansion methods and the exp function approach have been explored for discrete and fractional order PDEs as well.

The $\exp _{a}$ function method is a new and an efficient technique which has been acknowledged rapidly by the scholars. For example, Ali and Hassan, Hosseini et al., Zayed and Al-Nowehy all have utilized the exp a function method in [29],[30, 31] and [32] respectively. This paper aims to explore the exp a function approach to generate rational exponential solutions to conformable space-time fractional EW and the space-time fractional modified EW equations [33, 34].

The scheme of this article is as follows: In Sec. 2, the definition of conformable fractional derivative with some of its properties and the explanation of $\exp _{a}$ function approach are given. In Sec. 3, the conformable space-time FEW equations have been considered to elucidate via the above mentioned approach. The graphical representation of some solutions have also been given in the same section. Finally, a summarizing discussion of results and conclusion have been given in Sec. 4 \& 5 .

\section{Conformable fractional derivatives and the method of solutions}

Recall the Khalil's definition of the conformable derivative and with some properties.

Defination 1 Suppose $h: \mathbb{R}_{>0} \rightarrow \mathbb{R}$ be a function. Then, for all $t>0$,

$$
\left(T_{\alpha} h\right)(t)=\lim _{\varepsilon \rightarrow 0} \frac{h\left(t+\varepsilon t^{1-\alpha}\right)-h(t)}{\varepsilon}
$$

is known as the conformable fractional derivative of $h$ of order $\alpha, 0<\alpha \leq 1$. Some useful properties are being listed 
as follows:

$T_{\alpha}(a h+b g)=a\left(T_{\alpha} h\right)+b\left(T_{\alpha} g\right)$, for all $a, b \in \mathbb{R}$

$T_{\alpha}(h g)=h T_{\alpha}(g)+g T_{\alpha}(h)$

Let $h: \mathbb{R}_{>0} \rightarrow \mathbb{R}$ be a differentiable and $\alpha$-differentiable function, $g$ be a differentiable function defined in the range of $h$.

$$
T_{\alpha}(h \circ g)(t)=t^{1-\alpha} g^{\prime}(t) h^{\prime}(g(t)) .
$$

On the top of that, the following rules hold.

$T_{\alpha}\left(t^{\lambda}\right)=\lambda t^{\lambda-\alpha}$, for all $\lambda \in \mathbb{R}$

$T_{\alpha}(\lambda)=0$

$T_{\alpha}(h / g)=\frac{g\left(T_{\alpha} h\right)-h\left(T_{\alpha} g\right)}{g^{2}}$.

Conjointly, if $h$ is differentiable, then $T_{\alpha}(h)(t)=t^{1-\alpha} \frac{d h(t)}{d t}$.

\subsection{The $\exp _{a}$ function method}

The present subsection provides a concise explanation for the $\exp _{a}$ function method in generating new rational exponential solutions to nonlinear conformable space-time FEW and modified FEW equations. For this purpose, suppose that we have a nonlinear conformable time FDE that can be presented in the form

$$
F\left(u, D_{t}^{y} u, D_{x}^{\beta} u, D_{t}^{2 y} u, D_{x}^{2 \beta} u, \ldots\right)=0
$$

The FDE (1) can be changed into the following nonlinear ODE of integer order

$$
P\left(U, U^{\prime}, U^{\prime \prime}, \ldots,\right)=0
$$

with the use of following transformation

$$
u(x, t)=U(\xi), \xi=k \frac{x^{\beta}}{\beta}-l \frac{t^{y}}{y},
$$

where $k, l$ are nonzero arbitrary constants.

Let us try to search a non-trivial solution for the Eq. (2) in the following form

$$
U(\xi)=\frac{a_{0}+a_{1} a^{\xi}+\ldots+a_{N} a^{N \xi}}{b_{0}+b_{1} a^{\xi}+\ldots+b_{N} a^{N \xi}}
$$

where $a_{i}$ and $b_{i}$ for $(0 \leq i \leq N)$ are found later and $N$ is a free positive integer.

Replacing the Eq.(4) in the nonlinear Eq.(2), yields

$$
P\left(a^{\xi}\right)=q_{0}+q_{1} a^{\xi}+\ldots+q_{\tau} a^{\tau \xi}=0
$$

Setting $q_{i}(0 \leq i \leq \tau)$ in Eq.(4) to be zero, results in a set of nonlinear equations as below

$$
q_{i}=0, \quad i=0, \ldots, \tau
$$

which by solving the generated set (6), we approach to non-trivial solutions of the nonlinear FDE (1).

\section{Rational exponential solutions of space-time FMEW equation}

The space-time fractional modified equal-width equation [35], for finding its exact solutions via expa function method, is as follows:

$D_{t}^{y} u(x, t)+\epsilon D_{x}^{y} u^{3}(x, t)-\delta D_{x x t}^{3 y} u(x, t)=0, t>00<y \leq 1$,

Using the transformation (3), and integrating w.r.t. $\xi$ once, we get

$$
\delta l k^{2} U^{\prime \prime}-l U+\epsilon k U^{3}=0 .
$$

Through balancing, we select $N=1$, the nontrivial solution (4) reduces to:

$$
U(\xi)=\frac{\alpha_{1} \alpha^{\xi}+\alpha_{0}}{\beta_{1} \alpha^{\xi}+\beta_{0}}, \quad \alpha \neq 1
$$

By setting the above solution in reduced equation Eq. (8) and equating factors of each power of $a^{\xi}$ in the resulting equation, we reach a nonlinear algebraic set as

$\alpha_{0}^{3} k \epsilon-\alpha_{0} \beta_{0}^{2} l=0$,

$\alpha_{1} \beta_{0}^{2} \delta k^{2} l \log ^{2}(\alpha)-\alpha_{0} \beta_{0} \beta_{1} \delta k^{2} l \log ^{2}(\alpha)+3 \alpha_{0}^{2} \alpha_{1} k \epsilon-\alpha_{1} \beta_{0}^{2} l$

$-2 \alpha_{0} \beta_{0} \beta_{1} l=0$,

$\alpha_{0} \beta_{1}^{2} \delta k^{2} l \log ^{2}(\alpha)-\alpha_{1} \beta_{0} \beta_{1} \delta k^{2} l \log ^{2}(\alpha)+3 \alpha_{0} \alpha_{1}^{2} k \epsilon-\alpha_{0} \beta_{1}^{2} l$

$-2 \alpha_{1} \beta_{0} \beta_{1} l=0$,

$\alpha_{1}^{3} k \epsilon-\alpha_{1} \beta_{1}^{2} l=0$

which its solution yields

$$
\begin{aligned}
& \alpha_{1}=\mp \frac{\sqrt[4]{-\frac{\delta}{2}} \beta_{1} \sqrt{l \log (\alpha)}}{\sqrt{\epsilon}}, \alpha_{0}= \pm \frac{\sqrt[4]{-\frac{\delta}{2}} \beta_{0} \sqrt{l \log (\alpha)}}{\sqrt{\epsilon}}, \\
& k=-\frac{\sqrt{-2}}{\sqrt{\delta} \log (\alpha)} \alpha_{1}=\mp \frac{(-1)^{3 / 4} \beta_{1} \sqrt[4]{\delta} \sqrt{l \log (\alpha)}}{\sqrt[4]{2} \sqrt{\epsilon}} \\
& \alpha_{0}= \pm \frac{(-1)^{3 / 4} \beta_{0} \sqrt[4]{\delta} \sqrt{l \log (\alpha)}}{\sqrt[4]{2} \sqrt{\epsilon}}, k=\frac{\sqrt{-2}}{\sqrt{\delta} \log (\alpha)}
\end{aligned}
$$

Thus, the following new rational exponential solutions to the conformable space-time fractional modified EW equation can be written as

$$
U_{1,2}(\xi)=\frac{\sqrt[4]{-\frac{\delta}{2}} \sqrt{l \log (\alpha)}\left(\mp \beta_{1} \alpha^{\xi} \pm \beta_{0}\right)}{\sqrt{\epsilon}\left(\beta_{1} \alpha^{\xi}+\beta_{0}\right)}
$$

where $\xi=-\frac{\sqrt{-2}}{\sqrt{\delta} \log (\alpha)} \frac{x^{y}}{y}-l \frac{t^{y}}{y}$. The rational exponential solutions for different $y$ values and $\beta_{0}=\beta_{1}=\epsilon=\delta=l=1$ are graphed here.

$$
U_{3,4}(\xi)=\frac{(-1)^{3 / 4} \sqrt[4]{\delta} \sqrt{l \log (\alpha)}\left(\mp \beta_{1} \alpha^{\xi} \pm \beta_{0}\right)}{\sqrt[4]{2} \sqrt{\epsilon}\left(\beta_{1} \alpha^{\xi}+\beta_{0}\right)}
$$

where $\xi=\frac{\sqrt{-2}}{\sqrt{\delta} \log (\alpha)} \frac{x^{y}}{y}-l \frac{t^{y}}{y}$. 

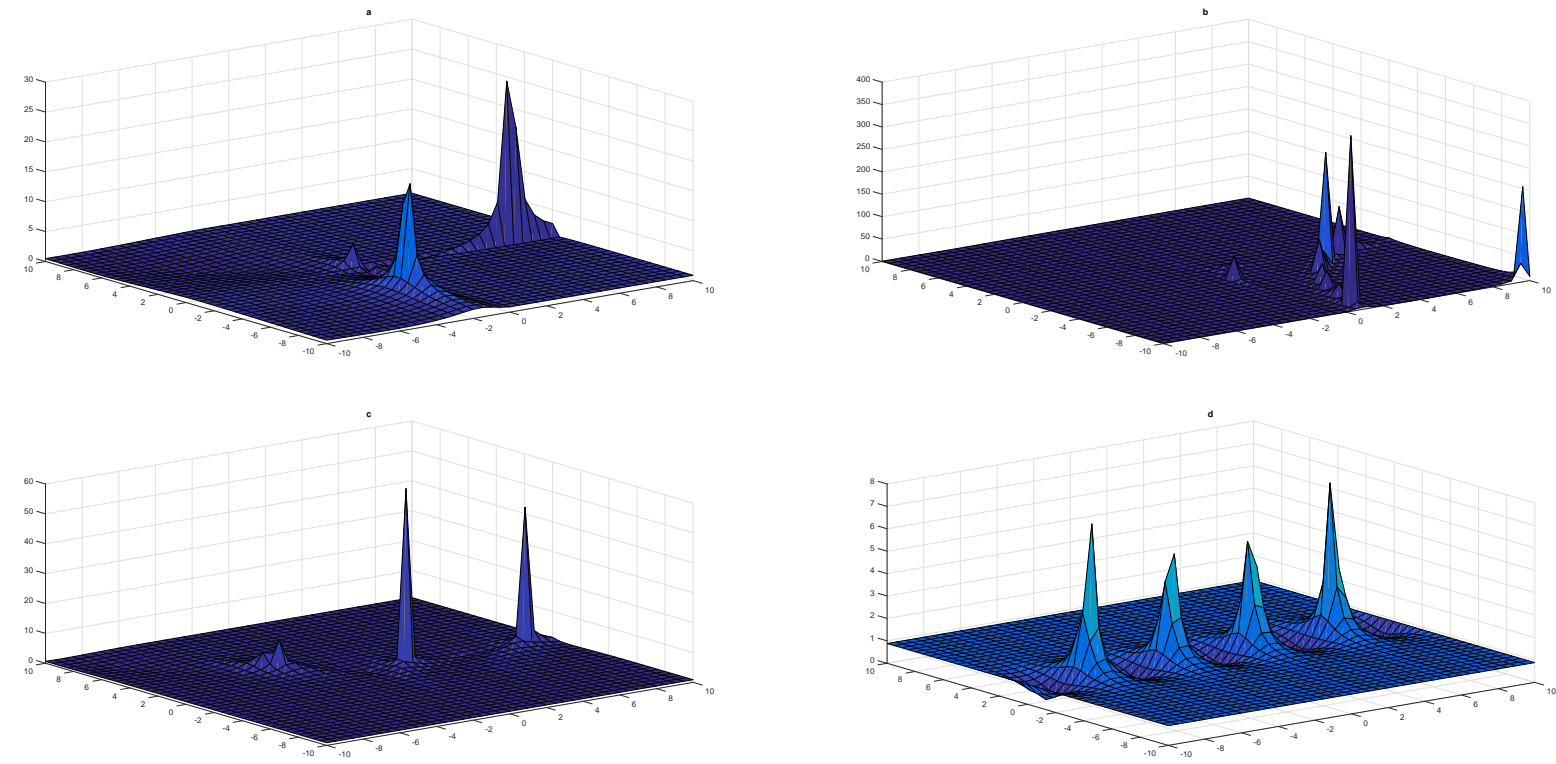

Fig. 1: Solution Profile of $u_{1,2}$ for $y=0.25,0.5,0.75$ and 1 respectively
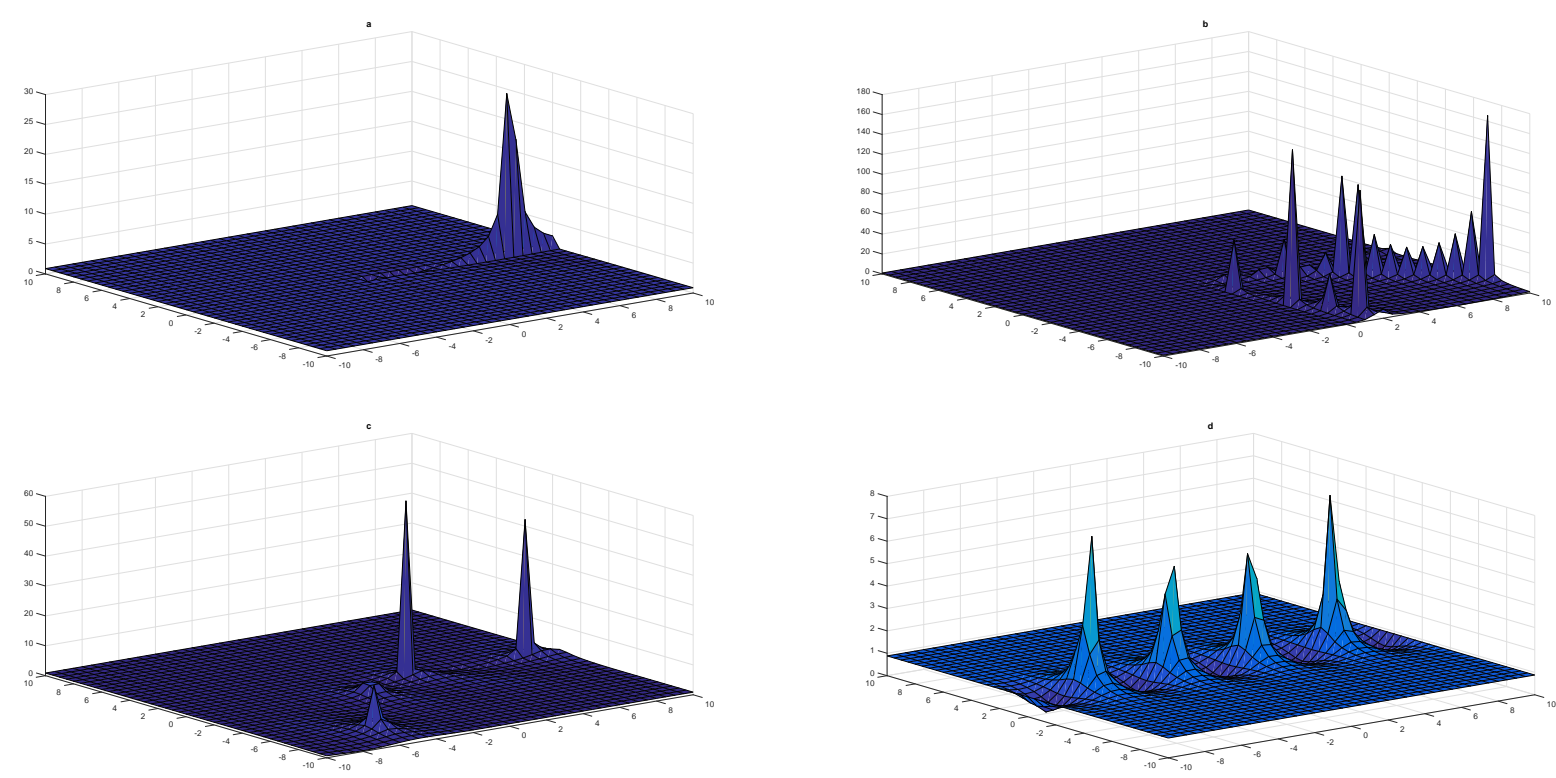

Fig. 2: Solution Profile of $u_{3,4}$ for $y=0.25,0.5,0.75$ and 1 respectively

\subsection{Rational exponential solutions of space-time FEW equation}

The conformable space-time fractional equal-width equation is as follows:

$D_{t}^{y} u(x, t)+\epsilon D_{x}^{y} u^{2}(x, t)-\delta D_{x x t}^{3 y} u(x, t)=0, t>00<y \leq 1$,
Using the transformation (3), and integrating w.r.t. $\xi$, we get

$$
\delta l k^{2} U^{\prime \prime}-l U+\epsilon k U^{2}=0 .
$$

Through balancing the terms we select $N=2$, the nontrivial solution (4) becomes

$$
U(\xi)=\frac{\alpha_{2} \alpha^{2 \xi}+\alpha_{1} \alpha^{\xi}+\alpha_{0}}{\beta_{2} \alpha^{2 \xi}+\beta_{1} \alpha^{\xi}+\beta_{0}}, \quad \alpha \neq 1
$$

By inserting the above non-trivial solution in reduced equation Eq. (13) and setting the coefficients of each power 

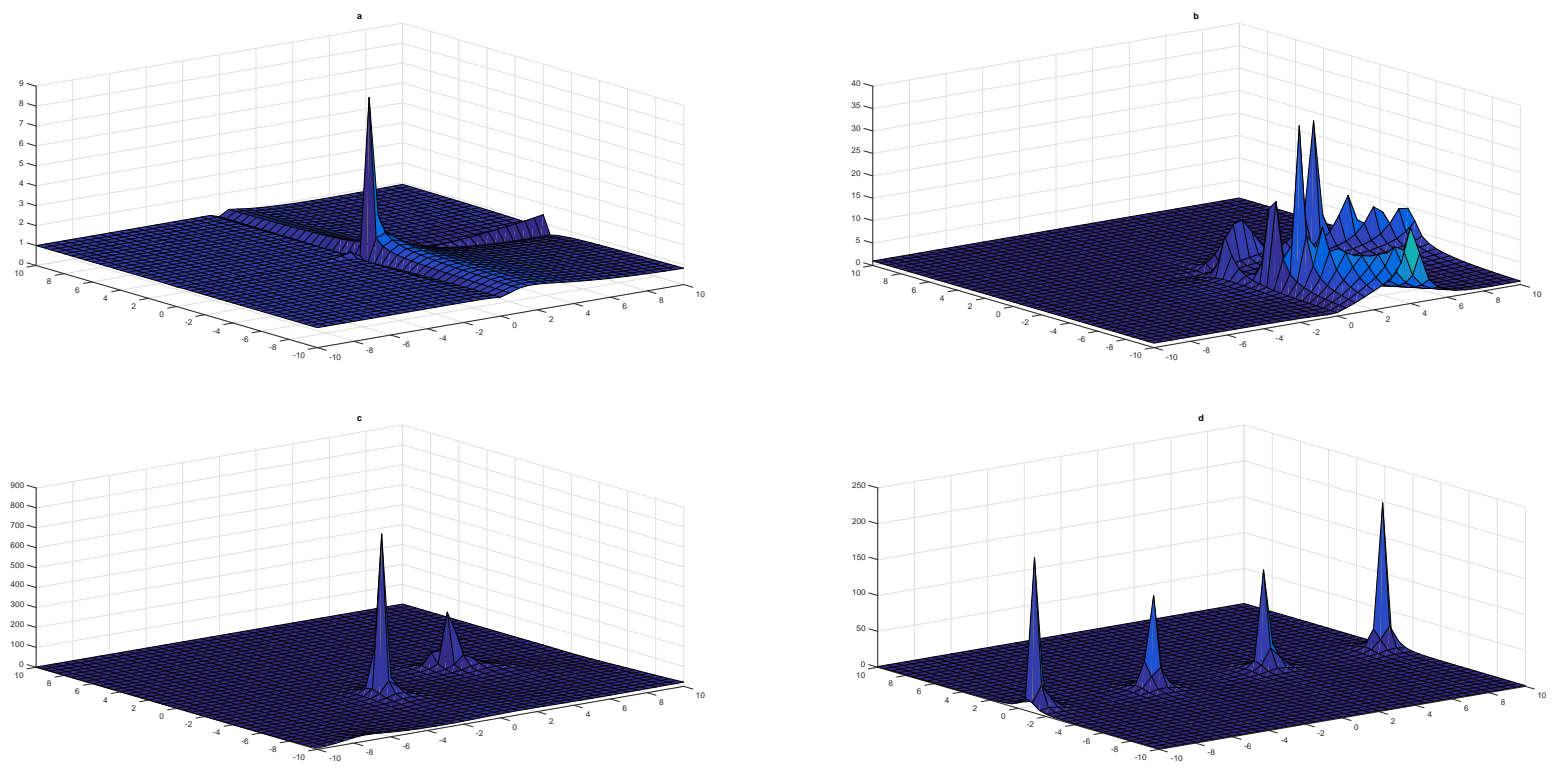

Fig. 3: Solution Profile of $u_{1}$ for $y=0.25,0.5,0.75$, and 1 respectively
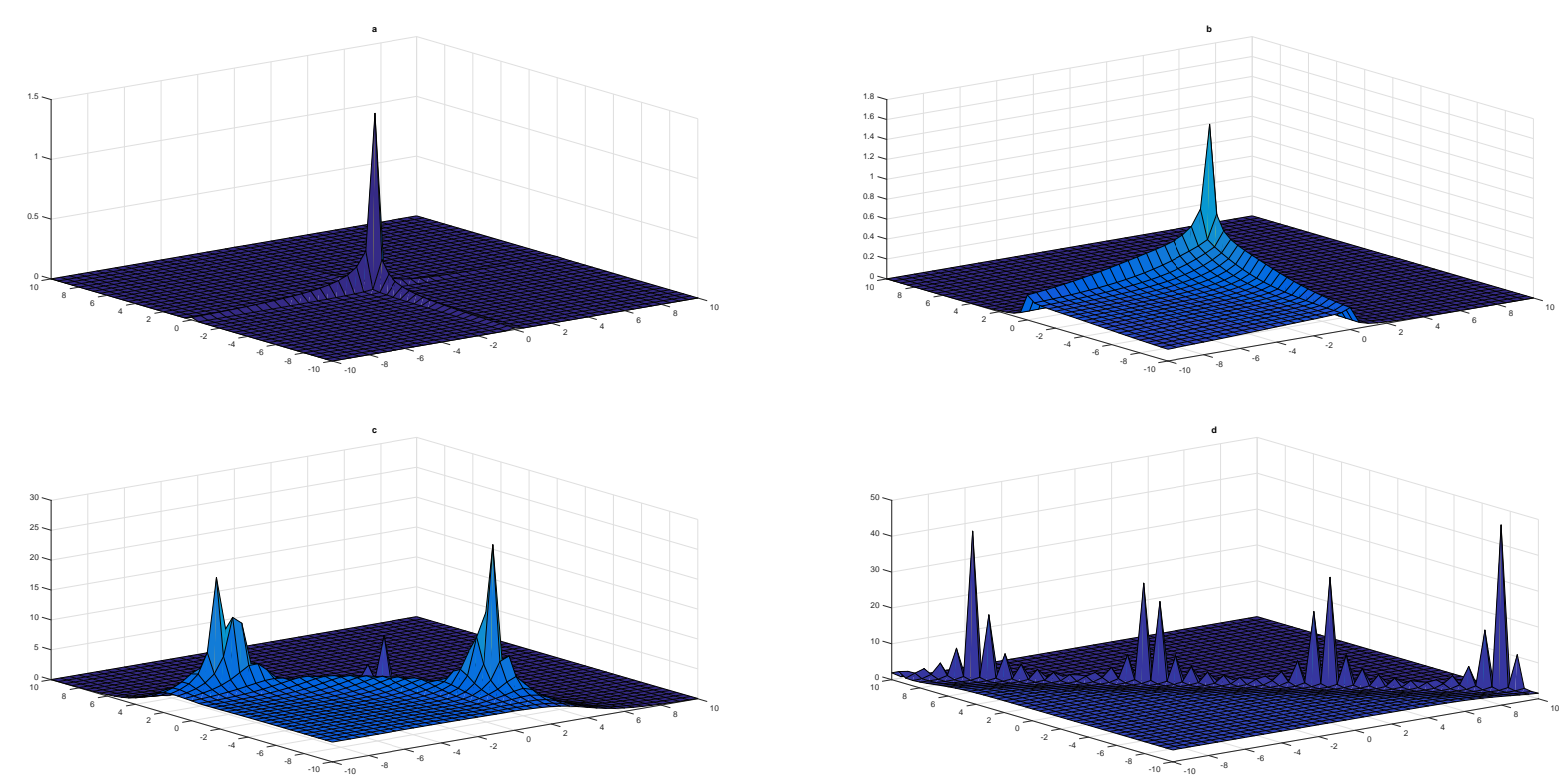

Fig. 4: Solution Profile of $u_{2}$ for $y=0.25,0.5,0.75$ and 1 respectively

of $\alpha^{\xi}$ in the resulting equation, we reach to a set of nonlinear algebraic equations as

$$
\begin{aligned}
& \alpha_{0}^{2} \beta_{0} k \epsilon-\alpha_{0} \beta_{0}^{2} l=0, \\
& \alpha_{1} \beta_{0}^{2} \delta k^{2} l \log ^{2}(\alpha)-\alpha_{0} \beta_{0} \beta_{1} \delta k^{2} l \log ^{2}(\alpha)+2 \alpha_{0} \alpha_{1} \beta_{0} k \epsilon \\
& +\alpha_{0}^{2} \beta_{1} k \epsilon-\alpha_{1} \beta_{0}^{2} l-2 \alpha_{0} \beta_{0} \beta_{1} l=0 \\
& 4 \alpha_{2} \beta_{0}^{2} \delta k^{2} l \log ^{2}(\alpha)+\alpha_{0} \beta_{1}^{2} \delta k^{2} l \log ^{2}(\alpha) \\
& -\alpha_{1} \beta_{0} \beta_{1} \delta k^{2} l \log ^{2}(\alpha)-4 \alpha_{0} \beta_{0} \beta_{2} \delta k^{2} l \log ^{2}(\alpha) \\
& +\alpha_{1}^{2} \beta_{0} k \epsilon+2 \alpha_{0} \alpha_{2} \beta_{0} k \epsilon+2 \alpha_{0} \alpha_{1} \beta_{1} k \epsilon+\alpha_{0}^{2} \beta_{2} k \epsilon
\end{aligned}
$$

$$
\begin{aligned}
& -\alpha_{2} \beta_{0}^{2} l-\alpha_{0} \beta_{1}^{2} l-2 \alpha_{1} \beta_{0} \beta_{1} l-2 \alpha_{0} \beta_{0} \beta_{2} l=0, \\
& 3 \alpha_{2} \beta_{0} \beta_{1} \delta k^{2} l \log ^{2}(\alpha)-6 \alpha_{1} \beta_{0} \beta_{2} \delta k^{2} l \log ^{2}(\alpha) \\
& +3 \alpha_{0} \beta_{1} \beta_{2} \delta k^{2} l \log ^{2}(\alpha)+2 \alpha_{1} \alpha_{2} \beta_{0} k \epsilon+\alpha_{1}^{2} \beta_{1} k \epsilon \\
& +2 \alpha_{0} \alpha_{2} \beta_{1} k \epsilon+2 \alpha_{0} \alpha_{1} \beta_{2} k \epsilon-\alpha_{1} \beta_{1}^{2} l-2 \alpha_{2} \beta_{0} \beta_{1} l \\
& -2 \alpha_{1} \beta_{0} \beta_{2} l-2 \alpha_{0} \beta_{1} \beta_{2} l=0, \\
& \alpha_{2} \beta_{1}^{2} \delta k^{2} l \log ^{2}(\alpha)+4 \alpha_{0} \beta_{2}^{2} \delta k^{2} l \log ^{2}(\alpha) \\
& -4 \alpha_{2} \beta_{0} \beta_{2} \delta k^{2} l \log ^{2}(\alpha)-\alpha_{1} \beta_{1} \beta_{2} \delta k^{2} l \log ^{2}(\alpha)+\alpha_{2}^{2} \beta_{0} k \epsilon \\
& +2 \alpha_{1} \alpha_{2} \beta_{1} k \epsilon+\alpha_{1}^{2} \beta_{2} k \epsilon+2 \alpha_{0} \alpha_{2} \beta_{2} k \epsilon-\alpha_{2} \beta_{1}^{2} l-\alpha_{0} \beta_{2}^{2} l
\end{aligned}
$$


$-2 \alpha_{2} \beta_{0} \beta_{2} l-2 \alpha_{1} \beta_{1} \beta_{2} l=0$,

$\alpha_{1} \beta_{2}^{2} \delta k^{2} l \log ^{2}(\alpha)-\alpha_{2} \beta_{1} \beta_{2} \delta k^{2} l \log ^{2}(\alpha)+\alpha_{2}^{2} \beta_{1} k \epsilon$

$+2 \alpha_{1} \alpha_{2} \beta_{2} k \epsilon-\alpha_{1} \beta_{2}^{2} l-2 \alpha_{2} \beta_{1} \beta_{2} l=0$,

$\alpha_{2}^{2} \beta_{2} k \epsilon-\alpha_{2} \beta_{2}^{2} l=0$.

which its solution yields

$$
\begin{aligned}
& \text { set }-1 \alpha_{0}=\mp \frac{i \beta_{0} \sqrt{\delta} l \log (\alpha)}{\epsilon}, \alpha_{1}= \pm \frac{2 i \beta_{1} \sqrt{\delta} l \log (\alpha)}{\epsilon}, \\
& \alpha_{2}=\mp \frac{i \beta_{1}^{2} \sqrt{\delta} l \log (\alpha)}{4 \beta_{0} \epsilon}, \beta_{2}=\frac{\beta_{1}^{2}}{4 \beta_{0}}, k= \pm \frac{i}{\sqrt{\delta} \log (\alpha)} \\
& \text { set }-2 \alpha_{0}=0, \alpha_{1}=\mp \frac{3 \beta_{1} \sqrt{\delta} l \log (\alpha)}{\epsilon}, \alpha_{2}=0, \\
& \beta_{2}=\frac{\beta_{1}^{2}}{4 \beta_{0}}, k=\mp \frac{1}{\sqrt{\delta} \log (\alpha)}
\end{aligned}
$$

Thus, from set-1 and set-2, the following new exact solutions to conformable space-time FEW equation can be written as

$$
U_{1}(\xi)=\frac{\iota \sqrt{\delta} l \log (\alpha)\left(\mp \frac{\beta_{1}^{2}}{4 \beta_{0} \epsilon} \alpha^{2 \xi} \pm \frac{2 \beta_{1}}{\epsilon} \alpha^{\xi} \mp \frac{\beta_{0}}{\epsilon}\right)}{\frac{\beta_{1}^{2}}{4 \beta_{0}} \alpha^{2 \xi}+\beta_{1} \alpha^{\xi}+\beta_{0}}
$$

where $\xi= \pm \frac{i}{\sqrt{\delta} \log (\alpha)} \frac{x^{y}}{y}-l \frac{t^{y}}{y}$. The rational exponential solutions for different $y$ values and $\beta_{0}=\beta_{1}=\epsilon=\delta=l=1$ are graphed here.

$$
U_{2}(\xi)=\frac{\mp \frac{3 \beta_{1} \sqrt{\delta} l \log (\alpha)}{\epsilon} \alpha^{\xi}}{\left(\frac{\beta_{1}^{2}}{4 \beta_{0}}\right) \alpha^{2 \xi}+\beta_{1} \alpha^{\xi}+\beta_{0}}
$$

where $\xi=\mp \frac{1}{\sqrt{\delta} \log (\alpha)} \frac{x^{y}}{y}-l \frac{l^{y}}{y}$.

\section{Discussion}

The conformable FEW and modified FEW equations are important in mathematical physics as a model for the simulation of one-dimensional wave transmission in nonlinear media with dispersion processes $[33,34]$. The conformable derivatives and the complex fractional transforms both are simple but effective to convert nonlinear FDE into a nonlinear ODE. The exp function approach for nonlinear FDEs with fractional complex transforms has its own benefits: direct, concise, and straightforward; and it can be used for many other nonlinear equations $[29,30]$. A series of new rational exponential solutions with their numerical simulation of conformable FEW equations has been accomplished through symbolic soft computation softwares.

\section{Conclusion}

The exact rational exponential solutions to conformable space-time FEW and modified FEW equations have been explored via $\exp _{a}$ function approach. The obtained solutions are entirely different from those given in [35]. These solutions are verified by inserting back in the reduced equations with the aid of symbolic computation in Mathematica. Furthermore, the numerical simulation of some solutions has been left for the reader to visualize them.

Acknowledgement: I would like to thank the anonymous referees for their expedient and esteemed comments and suggestions.

\section{References}

[1] G. Samko, A.A. Kilbas, and Marichev. Fractional Integrals and Derivatives: Theory and Applications. Gordon and Breach, Yverdon, 1993.

[2] A. Kilbas, M. H. Srivastava, and J. J. Trujillo. Theory ans application of fractional differential equations. North Holland Mathematics Studies, 2006, 204.

[3] R. Khalil, M. Al Horani, A. Yousef, and M. Sababheh. A new defination of fractional derivative. J.Comput.Appl.Math., 2014, 264, 65-70.

[4] Q. Zhou, M. Mirzazadeh, E. Zerrad, A. Biswas, and B. Milivoj. Bright, dark, and singular solitons in optical fibers withspatiotemporal dispersion and spatially dependent coefficients. Jornal of Modern Optics., 2016, 63(10), 427-430

[5] Kamyar Hosseini, Peyman Mayeli, and Reza Ansari. Bright and singular soliton solutions of the conformable time-fractional klein-gordon equations with different nonlinearities. Waves in Random and Complex Media, 2018, 28(3), 426-434.

[6] M. Kaplan and A. Bekir. The modified simple equation method for solving some fractional-order nonlinear equations. Pramana-J.Phys, 2016, 87(15).

[7] A. Biswas, Y. Yildirim, E. Yaser, H. Triki, A. S. Alshomrani, M. Zakh Ullah, Q. Zhou, S. P. Moshokoa, and M. Belic. Optical soliton perturbation with gerdjikov-ivanov equation by modified simple equation method. Optik, 2018, 157, 1235-1240.

[8] Y. Pandir, Y. Gurefe, and E. Misirli. The extended trial equation method for some time fractional differential equations. Discrete Dyn. Nat. Soc., 2013, 2013.

[9] A. Biswas, Y. Yildirim, E. Yaser, H. Triki, A.S. Alshomrani, M. Zakh Ullah, Q. Zhou, S. P. Moshokoa, and M. Belic. Optical soliton perturbation with full nonlinearity by trial equation method. Optik, 2018, 157, 1366-1375.

[10] B. Lu. The first integral method for some time fractional differential equations. J. Math. Anal. Appl., 2012, 395, 684-693.

[11] M. Eslami. Trial solution technique to chiral nonlinear schrodinger's equation in (1+2)-dimensions. Nonlinear Dynam, 2016, 85(2), 813-816. 
[12] M. Younis and Asim Zafar. Exact solutions to nonlinear differential equations of fractional order via $\left(\frac{G^{\prime}}{G}\right)$-expansion method. Appl. Math, 2014, 5, 1-6.

[13] D. Kumar, K. Hosseini, and F. Samadani. The sine- gordon expansion method to look for the traveling wave solutions of the tzitzéica type equations in nonlinear optics. Optik International Journal for Light and Electron Optics, 2017, 149, 439-446.

[14] K. Hosseini, D. Kumar, M. Kaplan, and E.Y. Bejarbaneh. New exact traveling wave solutions of the unstable nonlinear schrödinger equations. Commun. Theor. Phys., 2017, 68, 761-767.

[15] Y. Pandir, Y. Gurefe, and E. Misirli. A new approach to kudryashov's method for solving some nonlinear physical models. Int. J. Phys. Sci., 2012, 7,2860-2866.

[16] M. Kaplan, A. Bekir, and A. Akbulut. A generalized kudryashov method to some nonlinear evolution equations in mathematical physics. Nonlinear Dyn., 2016, 85, 2843-2850.

[17] E. Aksoy, A.C. Cevikel, and A. Bekir. Soliton solutions of (2+1)dimensional time-fractional zoomeron equation. Optik, 2016, 127, 6933-6942.

[18] K. Hosseini and R. Ansari. New exact solutions of nonlinear conformable time-fractional boussinesq equations using the modified kudryashov method. Waves Random Complex Media, 2017, 27, $628-636$.

[19] K. Hosseini, E. Yazdani Bejarbaneh, A. Bekir, and M. Kaplan. New exact solutions of some nonlinear evolution equations of pseudoparabolic type. Optical and Quantum Electronics, 2017, 49(7), 241.

[20] Z. Ayati, K. Hosseini, and M. Mirzazadeh. Application of kudryashov and functional variable methods to the strain wave equation in microstructured solids. Nonlinear Engineering, 2017, 6, 25-29.

[21] K. Hosseini, P. Mayeli, and D. Kumar. New exact solutions of the coupled sine- gordon equations in nonlinear optics using the modified kudryashov method. Journal of Modern Optics, 2018, 65(3), 361-364.

[22] K. Hosseini, F. Samadani, D. Kumar, and M. Faridi. New optical solitons of cubic-quartic nonlinear schrödinger equation. Optik, 2018, 157, 1101-1105.

[23] A. Bekir. Application of the extended tanh method for coupled nonlinear evolution equation. Commun. Nonlinear Sci., 2008, $13,1742-1751$.
[24] Bekir A., Adem C. Cevikel, Ö. Güner, and Sait San. Bright and dark soliton solutions of the $(2+1)$-dimenssional evolution equation. Mathematical Modelling and Analysis, 2014, 19, 118-126.

[25] E. Fan. Extended tanh-function method and its application to nonlinear equation. Phys. Lett. A., 2000, 277, 212-218.

[26] AM. Wazwaz. The extended tanh method for the zakharovkuznestsov(zk) equation, the modified zk equation, and its generalized forms. Commun. Nonlinear Sci., 2008, 13, 10391047.

[27] Kamyar Hosseini, Jalil Manafian, Farzan Samadani, Moham madreza Foroutan, Mohammad Mirzazadeh, and Qin Zhou. Resonant optical solitons with perturbation terms and fractional temporal evolution using improved $\tan (\phi(\eta) / 2)$ expansion method and exp function approach. Optik, 2018, 158, 933-939.

[28] S. Zhang. Application of exp-function to a kdv equation with variable-coefficients. Phys. Lett. A, 2007, 365, 448-453.

[29] A. T. Ali and E. R. Hassan. General $\exp _{a}$ function method for nonlinear evolution equations. Appl. Math. Comput., 2010, 217, 451-459.

[30] K. Hosseini, Z. Ayati, and R. Ansari. New exact solution of the tzitze ica type equations in nonlinear optics using the $\exp _{a}$ function method. J. Mod. Opt., 2018, 65(7), 847-851.

[31] K. Hosseini, A. Zabihi, F. Samadani, and R. Ansari. New explicit exact solutions of the unstable nonlinear schrödinger's equation using the $\exp _{a}$ and hyperbolic function methods. Optical and Quantum Electronics, 2018, 50(2), 82.

[32] E. M. E. Zayed and A. G. Al-Nowehy. Generalized kudryashov method and general $\exp _{a}$ function method for solving a high order nonlinear schrödinger equation. J. Space Explor., 2017, 6,1-26.

[33] Cary JR. Morrison PJ, Meiss JD. Scattering of regularized-longwave solitry waves. Physica D: Nonlinear Phenomena, 1984, 11(3), 324-336.

[34] Fan HL. The classiication of single trawling wave solutions to the generalized equal-width equation. Appl. Math. Comput., 2012, 219(2), 748-754.

[35] Alper Korkmaz. Exact solutions of space-time fractional ew and modified ew equations. Chaos, Solitons and Fractals, 2017, 96, 132-138. 